\title{
A Comparison of Two Excitation Modes for MEMS Electrothermal Displacement Sensors
}

\author{
Ali Mohammadi, Member, IEEE, S. O. Reza Moheimani, Fellow, IEEE, \\ and Mehmet Rasit Yuce, Senior Member, IEEE
}

\begin{abstract}
MEMS electrothermal displacement sensors can be operated in constant current (CC) or constant voltage (CV) excitation modes. The CV mode is more commonly used. However, there have been reports that the $\mathrm{CC}$ excitation mode may lead to a larger measured signal, and thus, it may be a better choice than the $\mathrm{CV}$ mode. In this letter, we present an analytic comparison of the two methods, and show that from a signalto-noise-ratio point of view, benefits of operating a sensor in $\mathrm{CC}$ mode are only marginal. The analytical investigation is supported by experiments performed on sensors integrated in a SOI-MEMS nanopositioner with low noise read out circuits, which leads to 0.04-nm $/ \sqrt{\mathrm{Hz}}$ displacement resolution for both excitation modes.
\end{abstract}

Index Terms-Micro-electromechancial systems (MEMS), electrothermal sensor, noise.

\section{INTRODUCTION}

$\mathbf{E}$ LECTROTHERMAL displacement sensors implemented in micro-electromechanical systems (MEMS) have attracted significant interest due to their small footprint, high sensitivity and straightforward integration in MEMS fabrication processes [1]. The electrothermal sensor shown in Fig. 1 comprises of a pair of resistors that detects the displacement of a moving stage [2]. The sensing mechanism is based on the heat conduction between the resistors and the stage. The sensitivity of the sensor is dominated by temperature gradient, i.e. a higher sensor temperature translates to a larger displacement to resistance variation gain. The thermal noise of the sensor increases with the temperature as well. Hence, the trade-off between sensitivity and low thermal noise determines the signal to noise ratio that translates to displacement resolution.

In order to heat up the resistors, electrical power is delivered either by a constant current (CC) [3] or a constant voltage (CV) source [4]. The CV mode is widely reported in the literature. However, the study reported in [3] indicated that the $\mathrm{CC}$ excitation leads to a higher sensitivity. Achieving higher resolution is critical for MEMS displacement sensors. In order

Manuscript received December 20, 2013; revised March 3, 2014; accepted March 10, 2014. Date of current version April 22, 2014. This work was supported by the Australian Research Council. The review of this letter was arranged by Editor A. Flewitt.

A. Mohammadi and S. O. R. Moheimani are with the School of Electrical Engineering and Computer Science, University of Newcastle, Newcastle, NSW 2308, Australia (e-mail: Reza.Moheimani@newcastle.edu.au).

M. R. Yuce is with the Department of Electrical and Computer Systems Engineering, Monash University, Clayton, VIC 3800, Australia.

Color versions of one or more of the figures in this letter are available online at http://ieeexplore.ieee.org.

Digital Object Identifier 10.1109/LED.2014.2313291

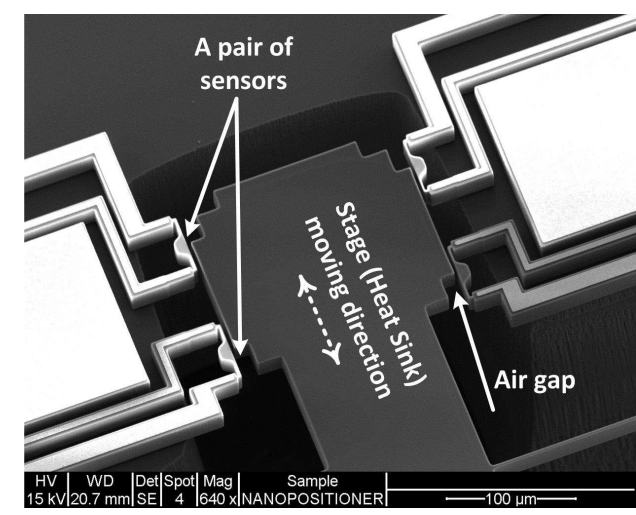

Fig. 1. Electrothermal displacement sensor fabricated in SOI MEMS.

to properly analyze this phenomenon, a new readout circuit that guarantees identical operating conditions for both $\mathrm{CV}$ and $\mathrm{CC}$ excitation modes is reported in this letter. A dynamic model of electrothermal sensors is presented to analytically compare the two approaches. Experimental results support the simulations that the achievable signal to noise ratio (SNR) is almost identical for both excitation modes.

\section{Constant Current Model}

The electrical powers delivered to the sensor with a constant voltage source $(V)$ and a constant current source $(I)$ are described by equations (1) and (2), respectively.

$$
\begin{aligned}
& P=V^{2} / R, \\
& P=R I^{2},
\end{aligned}
$$

Any displacement that results in the resistor temperature increase adds to its resistance value. According to (1), in the $\mathrm{CV}$ mode, this leads to a reduction in the electrical power dissipation, which results in a decrease in temperature. In comparison, (2) implies that any increase in the resistor temperature in CC mode leads to higher electrical power dissipation that translates to higher resistor temperature and this cycle makes the sensor more sensitive in CC mode.

A comprehensive model that addresses the dynamic behaviour of MEMS electrothermal systems is reported in [4], which explains the displacement $(x)$ functionality of the sensor resistance in the $\mathrm{CV}$ mode as depicted in Fig. 2. Here, $T_{T P}$ and $\mathrm{g}(\cdot)$ are the two main operators that relate the electrical power to temperature and the temperature to the sensor 


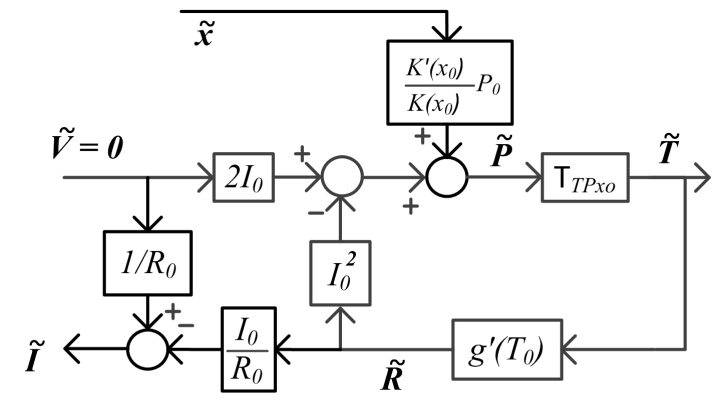

Fig. 2. Analytical model for the electrothermal sensor in CV mode [4].

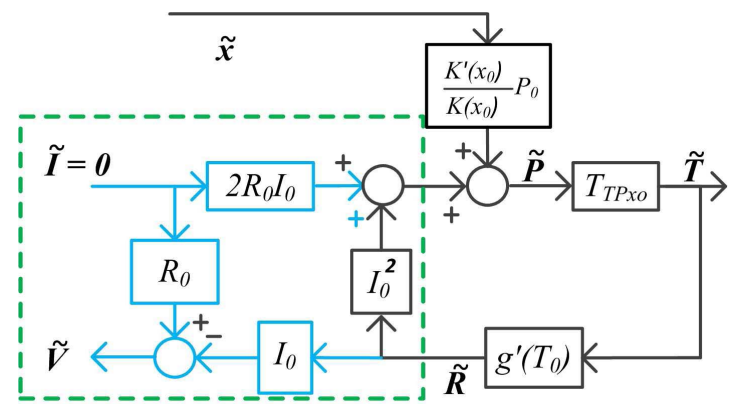

Fig. 3. Modified Model for the Electrothermal Sensor in CC Mode.

resistance, respectively. Also the operating point of the sensor is described by $V_{0}, I_{0}, R_{0}, P_{0}$ and $T_{0}$ coefficients in terms of its voltage, current, resistance, electrical power and temperature, respectively. The displacement perturbation $(\tilde{x})$, as the system input, results in temperature changes $(\tilde{T})$. This, in turn, leads to resistance fluctuations $(\tilde{R})$ that are measured by the output current variation $(\tilde{I})$. For small signal analysis, the Taylor series expansion of equation (1) can be used to linearize the nonlinear blocks around the operating point of the system:

$$
P=\frac{V_{0}^{2}}{R_{0}}+\frac{2 V_{0} \tilde{V} R_{0}-V_{0}^{2} \tilde{R}}{R_{0}^{2}}
$$

The resulting perturbation in electrical power $(\tilde{P})$ can be extracted from (3) by removing the constant term $\left(P_{0}=V_{0}^{2} / R_{0}\right)$ :

$$
\tilde{P}=2 I_{0} \tilde{V}-\tilde{R} I_{0}^{2}
$$

In order to adapt the model to the $\mathrm{CC}$ excitation mode, equation (2) is expanded as:

$$
P=R_{0} I_{0}^{2}+\tilde{R} I_{0}^{2}+2 R_{0} I_{0} \tilde{I}
$$

which leads to

$$
\tilde{P}=\tilde{R} I_{0}^{2}+2 R_{0} I_{0} \tilde{I}
$$

Thus, the CC mode model can be obtained by applying the electrical power signal in positive feedback to the system, as illustrated in Fig. 3. Note that constant coefficients, that match with the CC mode equations, are shown in the highlighted box.

Positive feedback typically leads to a larger gain. It often has a similar effect on noise. The signal transfer functions $T_{I x}$ and $T_{V x}$ and the noise transfer functions $T_{I n}$ and $T_{V n}$ for

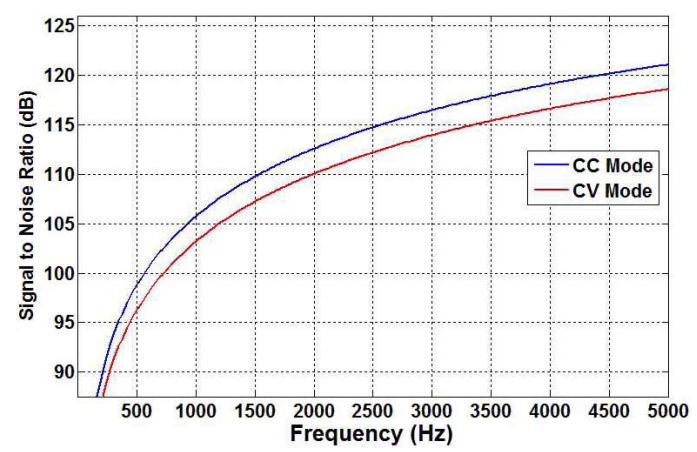

Fig. 4. The signal to noise ratio obtained by the analytical approach.

$\mathrm{CV}$ and $\mathrm{CC}$ modes, are obtained as:

$$
\begin{aligned}
T_{\tilde{I} \tilde{x}_{x_{0}}} & =\frac{K^{\prime}\left(x_{0}\right)}{K\left(x_{0}\right)}\left(\frac{-I_{0}}{R_{0}} \frac{g^{\prime}\left(T_{0}\right) P_{0} T_{T P_{x_{0}}}}{1+I_{0}^{2} g^{\prime}\left(T_{0}\right) T_{T P_{x_{0}}}}\right) \\
T_{\tilde{V} \tilde{x}_{x_{0}}} & =\frac{K^{\prime}\left(x_{0}\right)}{K\left(x_{0}\right)}\left(\frac{-I_{0}}{R_{0}} \frac{g^{\prime}\left(T_{0}\right) P_{0} T_{T P_{x_{0}}}}{1-I_{0}^{2} g^{\prime}\left(T_{0}\right) T_{T P_{x_{0}}}}\right) \\
T_{\tilde{I} n_{R x_{0}}} & =\frac{-I_{0} / R_{0}}{1+I_{0}^{2} g^{\prime}\left(T_{0}\right) T_{T P_{x_{0}}}} \\
T_{\tilde{V} n_{R x_{0}}} & =\frac{-I_{0} / R_{0}}{1-I_{0}^{2} g^{\prime}\left(T_{0}\right) T_{T P_{x_{0}}}}
\end{aligned}
$$

where $K(x)$ determines the $T_{T P}$ gain as a function of displacement $x$, and $n_{R}$ is the sensor noise. The power spectral density of the net resistance noise is the sum of the thermal noise $\left(n_{R_{j}}\right)$ and the flicker noise $\left(n_{R_{f}}\right)$ as:

$$
S_{n_{R}}(f)=S_{n_{R_{j}}}(f)+S_{n_{R_{f}}}(f)=\frac{4 k_{B} T_{0} R_{0}^{2}}{P_{0}}+\frac{\alpha R_{0}^{2}}{f N_{c a r r}}
$$

where $k_{B}$ and $f$ represent the Boltzmann constant and frequency, respectively and $\alpha$ (the Hooge factor) and $N_{\text {carr }}$ are experimentally determined. The signal to noise ratio (SNR) for $\mathrm{CV}$ and CC modes is defined by equations (12) and (13) over a desired frequency bandwidth.

$$
\begin{aligned}
& S N R_{C V}=20 \log \left(\frac{\sqrt{\int\left|T_{\tilde{I} \tilde{x}_{x_{0}}}(f)\right|^{2} d f}}{\sqrt{\int\left|T_{\tilde{I} n_{R_{x_{0}}}}(f)\right|^{2} S_{n_{R}}(f) d f}}\right) \\
& S N R_{C C}=20 \log \left(\frac{\sqrt{\int\left|T_{\tilde{V} \tilde{x}_{x_{0}}}(f)\right|^{2} d f}}{\sqrt{\int\left|T_{\tilde{V} n_{R_{x_{0}}}}(f)\right|^{2} S_{n_{R}}(f) d f}}\right)
\end{aligned}
$$

The SNR values corresponding to (12) and (13) were evaluated and plotted in Fig. 4. It can be observed that the CC excitation mode leads to a slightly higher-about $2 \mathrm{~dB}-\mathrm{SNR}$. The $5 \mathrm{kHz}$ simulation frequency bandwidth covers the typical electrothermal sensor bandwidth [4]. Also further simulations showed that the empirically defined values such as $N_{\text {carr }}$ and $\alpha$ affect the absolute noise values but the differences are the same. Bandwidth of the displacement sensor is also determined by the thermoelectric feedback loop [5]. In particular, assuming a first order transfer function for $T_{T P}$, equations (7) and (8) imply that operating the sensor in the CV mode leads to a higher sensor bandwidth. 


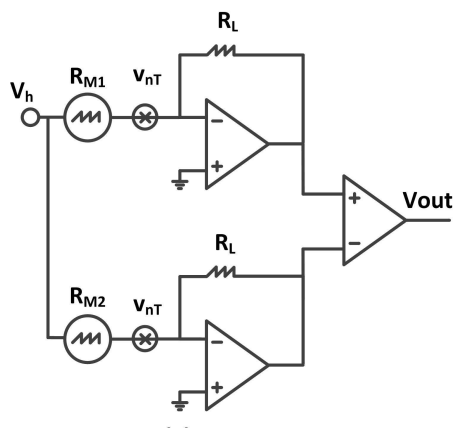

(a)

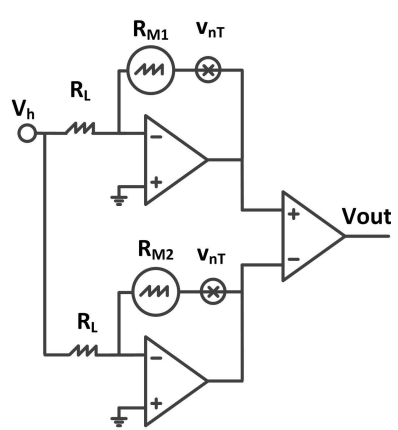

(b)
Fig. 5. Electrothermal sensor readout circuits: a) $\mathrm{CV}$ and b) CC.

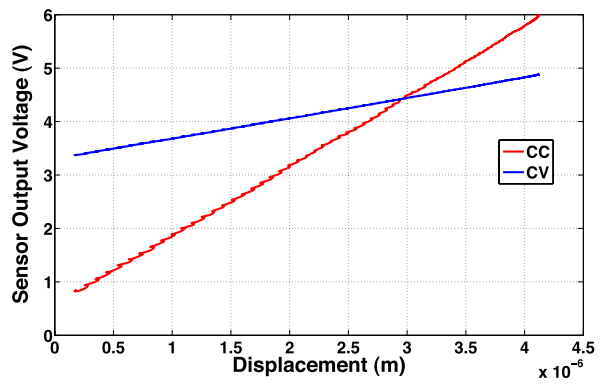

Fig. 6. Sensitivity in CC and CV modes.

\section{EXPERIMENTAL SET-UP AND RESUlts}

Experiments were performed on a SOI-MEMS 2-Degree-offreedom (2-DOF) nanopositioner with integrated eletrothermal sensors [2]. The width, length and height of each sensor are $2 \mu \mathrm{m}, 50 \mu \mathrm{m}$ and $25 \mu \mathrm{m}$, respectively, which translates to around $300 \mathrm{ohms}$ heated electrical resistance $\left(R_{0}\right)$ for a typical bias current of $\left(I_{0}=16 \mathrm{~mA}\right) . G(\cdot)$ and $T_{T P}$ transfer functions as well as frequency responses corresponding to (7) to (10) were identified based on the procedure proposed in [4].

A readout circuit was designed and tested to verify the analytic model and simulations. In the conventional $\mathrm{CV}$ mode [Fig. 5-(a)] the first pair of operational amplifiers acts as a transimpedance amplifier that converts the current variations arising from differential resistive changes to a voltage output. $V_{h}, R_{M}$ and $v_{n T}$ represent the heating bias voltage, the MEMS sensor resistor and the sensor noise, respectively. The subsequent differential amplifier produces a voltage output $\left(V_{\text {out }}\right)$ which is a linear function of the resistive changes. In order to guarantee equal readout circuit noise contributions, a similar configuration is designed for the CC mode excitation by only swapping the feedback resistor $\left(R_{L}\right)$ with the MEMS resistor as depicted in Fig. 5-(b).

During experiments, the heat-sink (stage) shown in Fig. 1 was actuated by an electrostatic comb drive. The sensor output voltage is measured versus the stage displacement by an optical microsystem analyzer (MSA-050-3D), as illustrated in Fig. 6. The sensitivity is clearly higher in $\mathrm{CC}$ mode $(1.3 \mathrm{~V} / \mu \mathrm{m})$ compared with the $\mathrm{CV}$ mode $(0.38 \mathrm{~V} / \mu \mathrm{m})$. However, the achievable displacement resolution is determined by the sensitivity and output voltage noise floor. Since the amplifiers have identical noise contributions, the output power spectrum can be used to compare the achievable SNRs

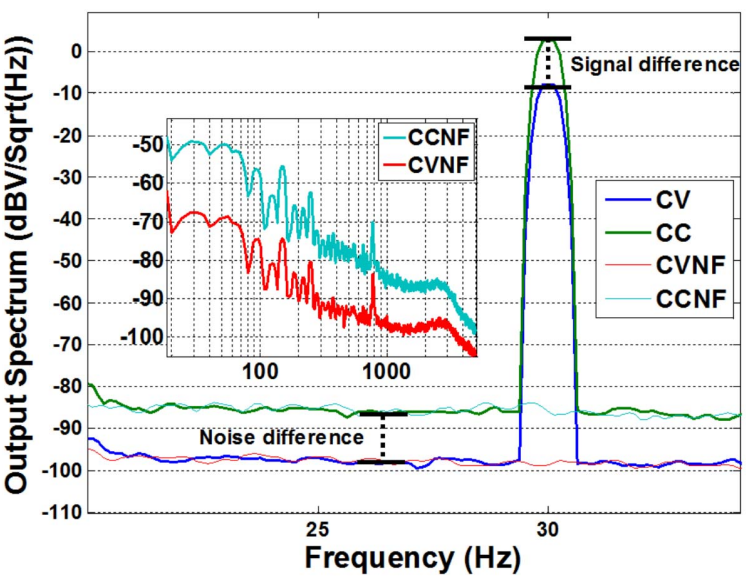

Fig. 7. Measured output signal and noise floor power spectrum. CCNF and CVNF represent the noise floor in constant current and constant voltage modes, respectively.

corresponding to the two modes. The stage is actuated by a $30 \mathrm{~Hz}$ sinusoidal signal and the sensor output power spectrum was measured as illustrated in Fig. 7. The CC excitation results in approximately $10 \mathrm{~dB}$ higher signal level. It is observed that the noise floor (NF) has also risen by about the same amount, which is in close agreement with the analysis. The measured noise floor and sensitivity translates to $0.04 \mathrm{~nm} / \sqrt{\mathrm{Hz}}$ displacement resolution for both cases. The wide band noise is also measured over the entire sensor bandwidth. As illustrated by the inset in Fig. 7, the noise floor corresponding to the $\mathrm{CC}$ is higher than $\mathrm{CV}$, which agrees with the simulation results presented in Fig. 4. The small difference between the simulations and experimental results is most likely due to the approximations used in the analytical modelling particularly for the identification of the physical attributes of the silicon.

\section{CONCLUSION}

The noise performance of a MEMS electrothermal displacement sensor excited by a constant current (CC) and a constant voltage $(\mathrm{CV})$ source was investigated. The analytical model shows that the achievable SNR in the CC mode is slightly higher (about $2 \mathrm{~dB}$ ). The experiments have been carried out on electrothermal sensors integrated in a 2-DoF a SOI-MEMS nanopositioner. The measurements show that although the CC mode leads to a higher output signal level, it increases the noise floor as well. Hence, the maximum achievable SNR and displacement resolution by the two modes is almost identical, which is in close agreement with the analytical result.

\section{REFERENCES}

[1] M. A. Lantz et al., "A micromechanical thermal displacement sensor with nanometer resolution," Nanotechnology, vol. 16, no. 8, pp. 1089-1094, 2005.

[2] A. Mohammadi et al., "A feedback controlled MEMS nanopositioner for on-chip high-speed AFM," J. Microelectromech. Syst., 2013, doi: 10.1109/JMEMS.2013.2287506.

[3] B. Krijnen et al., "A single-mask thermal displacement sensor in MEMS," J. Micromech. Microeng., vol. 21, no. 7, p. 074007, 2011.

[4] A. Sebastian and D. Wiesmann, "Modeling and experimental identification of silicon microheater dynamics: A systems approach," J. Microelectromech. Syst., vol. 17, no. 4, pp. 911-920, 2008.

[5] U. Durig, "Fundamentals of micromechanical thermoelectric sensors," J. Appl. Phys., vol. 98, no. 4, pp. 044906-1-044906-14, 2005. 